low risk of developing cardiovascular disease; people who had ever taken lipid-lowering therapy or had undergone revascularization were excluded. In a 5:2 ratio, participants were randomly assigned $40 \mathrm{mg}$ rosuvastatin or placebo. All participants received therapeutic lifestyle intervention counseling. Intima-media thickness was assessed by carotid ultrasonography before randomization and at 6 , 12, 18 and 24 months.

From baseline, rosuvastatin significantly improved patients' lipid profiles compared with placebo. Overall and in individual carotid segments, atherosclerosis progression was significantly slowed in rosuvastatin-treated patients compared with placebo-treated patients (all $P<0.001$ ); however, rosuvastatin treatment was not associated with significant regression, except in the common carotid artery $(P=0.004)$. Progression was seen in all carotid segments in placebo-treated patients, but not in rosuvastatin-treated patients. Few serious adverse events were reported, with similar rates in both treatment groups.

The $40 \mathrm{mg}$ dose selected was chosen to maximize efficacy, but is not a recommended starting dose. The authors suggest that larger, longer-term studies are done to determine the implications of these findings.

Original article Crouse JR III et al. (2007) Effect of rosuvastatin on progression of carotid intima-media thickness in low-risk individuals with subclinical atherosclerosis: the METEOR trial. JAMA 297: 1344-1353

\section{Fracture risk is increased in patients with atrial fibrillation or receiving amiodarone}

Cardiac arrhythmias (atrial fibrillation being the most common) are mainly seen in the elderly, in whom osteoporotic fractures are also common. Whether associations exist between cardiac arrhythmias and the risk of fractures-caused by the heart disease itself or by adverse effects of antiarrhythmic drugs-is unclear. Rejnmark and colleagues conducted a population-based case-control study to investigate whether any such relationships can be identified.

The authors identified from Danish registries 124,655 individuals (mean age 43 years) who had sustained a fracture during 2000, and 373,962 controls matched for age and sex. Data on the purchase of any antiarrhythmic drug was gathered from a national prescription database. Confounding diseases and the use of any other drugs thought to affect fracture risk were also identified.

In total, $27 \%$ of the fractures were sustained at the hip, spine or forearm. Crude and adjusted fracture risk was significantly raised by a diagnosis of atrial fibrillation (30\% and $14 \%$ rise), and by amiodarone therapy ( $80 \%$ and $47 \%$ rise). Although the crude fracture risk was raised slightly (8\%) in patients receiving digoxin, after adjustment the risk was reduced by $25 \%$; risk of any, hip, or forearm fracture fell with increasing dose. Drug treatment only affected fracture risk in individuals aged $>65$ years. Fracture risk was not affected by sex, former antiarrythmic drug use, or other tachyarrhythmias.

The authors suggest that amiodarone treatment and atrial fibrillation are risk factors for fracture, especially in the elderly population.

Original article Rejnmark L et al. (2007) Fracture risk in patients treated with amiodarone or digoxin for cardiac arrhythmias: a nation-wide case-control study. Osteoporosis Int 18: 409-417

\section{Hydrochlorothiazide has a beneficial and durable effect on BMD in postmenopausal women}

It is thought that thiazide diuretics might prevent osteoporosis by reducing renal calcium secretion. Bolland and colleagues previously conducted a 2-year controlled, blind study in postmenopausal women who received hydrochlorothiazide $50 \mathrm{mg}$ daily or placebo. There were significant, sustained, between-group differences in the BMD of the leg, forearm and overall body. The authors have now completed a 2-year extension of this study, with a view to establishing whether the effects of hydrochlorothiazide have long-term durability.

Out of 138 participants who completed the initial trial, 122 women continued with the extension phase on the same treatment regime (active treatment, $n=57$; placebo, $n=65$ ). BMD was measured at half-yearly intervals.

BMD tended to be greater at all sites in the treatment group than in the placebo group, with between-group differences at 4 years ranging from $0.4 \%$ to $1.4 \%$; the differences remained stable after initial divergence between months 6 and 12. Significant differences were observed 